\title{
Excitation Rate and Background Measurements During LIF Studies on Krypton
}

C. A. Whitehead

B. D. Cannon

J. F. Wacker

April 1993

Prepared for the U.S. Department of Energy under Contract DE-AC06-76RLO 1830

Pacific Northwest Laboratory Operated for the U.S. Department of Energy by Battelle Memorial institute 


\title{
DISCLAIMER
}

This report was prepared as an account of work sponsored by an agency of the United States Government. Neither the United States Government nor any agency thereof, nor Battelle Memorial Institute, nor any of their employees, makes any warranty, expressed or implied, or assumes any legal liability or responsibility for the accuracy, completeness, or usefulness of any information, apparatus, product, or process disclosed, or represents that its use would not infringe privately owned rights. Reference herein to any specific commercial product, process, or service by trade name, trademark, manufacturer, or otherwise does not necessarily constitute or imply its endorsement, recommendation, or favoring by the United States Government or any agency thereof, or Battelle Memorial Institute. The views and opinions of authors expressed herein do not necessarily state or reflect those of the United States Government or any agency thereof.

\author{
PACIFIC NORTHWEST LABORATORY \\ operated by \\ BATTELLE MEMORIAL INSTITUTE \\ for the \\ UNITED STATES DEPARTMENT OF ENERGY \\ under Contract DE-ACO6-76RLO 1830
}

Printed in the United States of America

Available to DOE and DOE contractors from the

Office of Scientific and Technical Information, P.O. Box 62, Oak Ridge, TN 37831; prices available from (615) 576-8401. FTS 626-8401.

Available to the public from the National Technical Information Service, U.S. Department of Commerce, 5285 rort Royal Rd., Springfield, VA 22161. 
Excitation Rate and Background Measurements During LIF Studies on Krypton

C. A. Whitehead

B. D. Cannon

J. F. Wacker

April 1993

Interim Report to $\mathrm{DOE} / \mathrm{AN}-10$

Prepared for

the U.S. Department of Energy

under Contract DE-AC06-76RLO 1830

Pacific Northwest Laboratory

Richland, Washington 99352 


\section{EXECUTIVE SUMMARY}

The Krypton Isotope Laser Analysis (KIIA) method is being developed at the Pacific Northwest Laboratory (PNL) to measure ${ }^{85} \mathrm{Kr}$ concentrations in small air samples. The technique uses high-resolution lasers to excite individual isotopes of krypton specifically to induce ${ }^{85} \mathrm{Kr}$ to fluoresce for detection by optical means. The first step of the KILA method, involving the excitation of krypton atoms into a long-lived metastable state, has been shown to be feasible. Production of krypton metastables via two-photon excitation to the $2 \mathrm{p}_{6}$ state has been shown to be $0.15 \%$ efficient in $0.13 \mathrm{mTorr}$ of krypton-sufficiently high to demonstrate overall feasibility of the KIlLA method. Since this goal was met, focus has been directed toward development of a working vacuum ultraviolet(VUV) fluorescence detection system and toward understanding the VUV background. This report describes the progress made in these two areas.

The second step of the KILA process is to optically pump all except the ${ }^{85} \mathrm{Kr}$ isotopes from the metastabie state back to the ground state using laser-induced fluorescence(LIF). The rate of this process and the VUV background afterward will determine the sensitivity and selectivity of the KILA approach. De-excitation of the metastable population was accomplished via one-photon absorption of a continuous-wave(cw) laser to the $2 \mathrm{p}_{8}$ energy level, which is an efficient optical pumping route to the ground state. Non-isotopically selective de-excitation rates as high as $5 \times 10^{5}$ $\mathrm{sec}^{-1}$ have been measured, yielding a signal-to-background ratio of $>10^{6}$. The lifetime of the metastables is $1.2 \mathrm{msec}$ in $200 \mathrm{~m}$ Torr of neon-much longer than the time required to de-excite krypton metastables and to detect fluorescence produced by ${ }^{85} \mathrm{Kr}$. After attaining these high deexcitation rates, a gated VUV detection system was built with a dynamic range large enough to measure a small background following de-excitation of large metastable populations. Future experiments will focus on reducing the background level by another 2-3 orders of magnitude and perfecting the isotopically selective de-excitation technique with samples of known isotopic abundances. 


\section{CONTENTS}

EXECUTIVE SUMMARY $\ldots \ldots \ldots \ldots \ldots \ldots \ldots \ldots \ldots \ldots \ldots \ldots \ldots \ldots \ldots$ iii

INTRODUCTION $\ldots \ldots \ldots \ldots \ldots \ldots \ldots \ldots \ldots \ldots \ldots \ldots \ldots \ldots \ldots \ldots \ldots$

EXPERIMENTAL METHODS $\ldots \ldots \ldots \ldots \ldots \ldots \ldots \ldots \ldots \ldots \ldots \ldots \ldots \ldots \ldots \ldots$

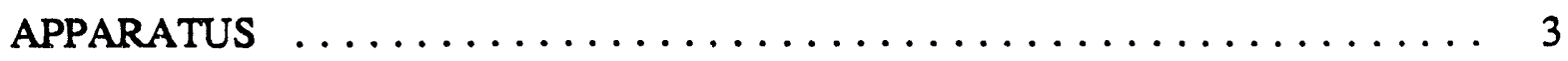

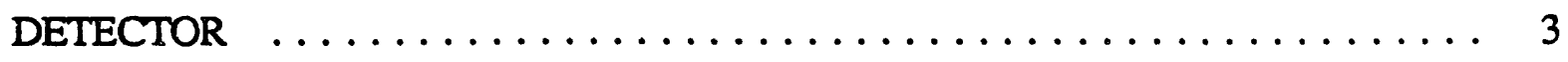

ELECTRONICS $\ldots \ldots \ldots \ldots \ldots \ldots \ldots \ldots \ldots \ldots \ldots \ldots \ldots \ldots \ldots \ldots \ldots \ldots \ldots$

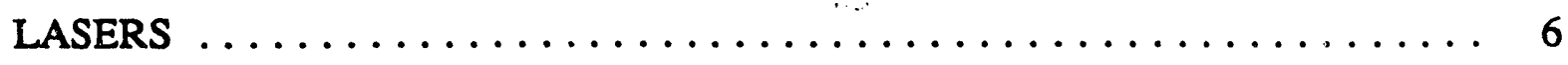

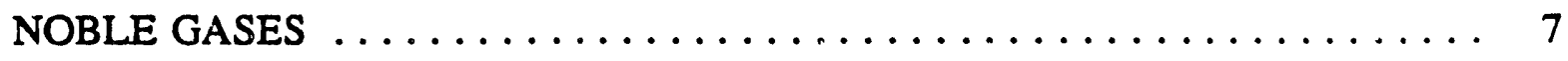

METASTABLE PRODUCTION $\ldots \ldots \ldots \ldots \ldots \ldots \ldots \ldots \ldots \ldots \ldots \ldots$

METASTABLE DE-EXCITATION $\ldots \ldots \ldots \ldots \ldots \ldots \ldots \ldots \ldots \ldots \ldots \ldots \ldots \ldots \ldots$

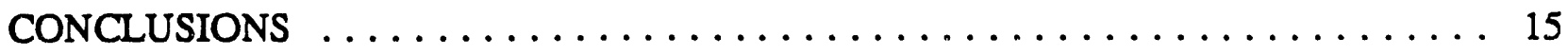

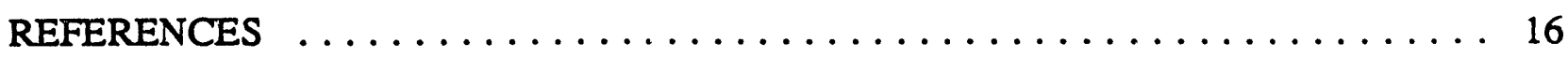

DISTRIBUTION LIST $\ldots \ldots \ldots \ldots \ldots \ldots \ldots \ldots \ldots \ldots \ldots \ldots \ldots \ldots \ldots \ldots \ldots \ldots \ldots$ 


\section{FIGURES}

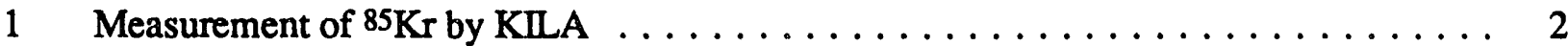

2 Apparatus for Detecting VUV Fluorescence from $\mathrm{Kr}$ Metastables . . . . . . . . . . . 4

3 Energy-level Diagram of Excitation/De-excitation Scheme . . . . . . . . . . 5

4 Time-resolved VUV Fluorescence from $1 s_{4}$ Level $\ldots \ldots \ldots \ldots \ldots \ldots \ldots$

5 Plot of De-excitation Rate vs. Buffer Gas Pressure $\ldots \ldots \ldots \ldots \ldots \ldots \ldots \ldots$

6 Ploi of Background vs. Buffer Gas Pressure $\ldots \ldots \ldots \ldots \ldots \ldots$ 


\section{INTRODUCTION}

The analysis of ${ }^{85} \mathrm{Kr}$ concentrations in air samples provides important information about nuclear-related activities and represents an area of considerable interest. An important goal is the development of a method to measure $<10^{6}{ }^{85} \mathrm{Kr}$ atoms in the presence of $>10^{10}$ times quantities of more stable krypton. Conventional beta-counting has the selectivity to measure ${ }^{85} \mathrm{Kr}$ at these levels, but samples of at least $10^{7}$ to $10^{8}$ atoms are required due to the low sensitivity of this method. Mass spectrometric techniques can in principle detect as few as 1000 atoms but cannot measure isotope ratios lower than 10-9. In contrast, laser-based spectrometric methods have both high sensitivity and selectivity, thereby offering the potential of measuring ${ }^{85} \mathrm{Kr}$ in liter-sized air samples.

At Pacific Northwest Laboratory (PNL), (a) we are developing a laser-based method to measure ambient ${ }^{85} \mathrm{Kr}$ in one liter of air. The method, called $\mathrm{KILA}$ (Krypton Isotope Laser Analysis), uses high-resolution lasers to excite individual isotopes of krypton specifically to induce ${ }^{85} \mathrm{Kr}$ to fluoresce for detection by optical means. The KIlA method, described in Figure 1, consists of three steps. In the first step, krypton atoms are excited into a long-lived metastable state using non-isotopically selective excitation. In the second step, all isotopes except ${ }^{85} \mathrm{Kr}$ are selectively de-excited back into the ground state using laser-induced fluorescence(LIF). The LIF process uses lasers tuned to hyperfine transitions of specific $\mathrm{Kr}$ isotopes. Each metastable emits a vacuum ultraviolet photon during de-excitation. Only ${ }^{85} \mathrm{Kr}$ metastables remain in significant abundance at the end of this step. In the third step, the ${ }^{85} \mathrm{Kr}$ metastables are selectively de-excited, and the resulting vacuum-ultraviolet (VUV) fluorescence is measured using a suitable detector. The development of this method requires

- efficient production of krypton metastables

- isotopically selective de-excitation of krypton metastables

- a detector to measure the VUV fluorescence produced by de-excitation of $\mathrm{Kr}$ metastables - demonstration of the detection of ${ }^{85} \mathrm{Kr}$ atoms in a liter of normal air.

This report describes progress on lowering the VUV background to a level useful for trace isotope analysis and on the development of a suitable VUV detection system.

(a) Operated for the U.S. Department of Energy by Battelle Memorial Institute under Contract DE-AC06-76RL0 1830. 


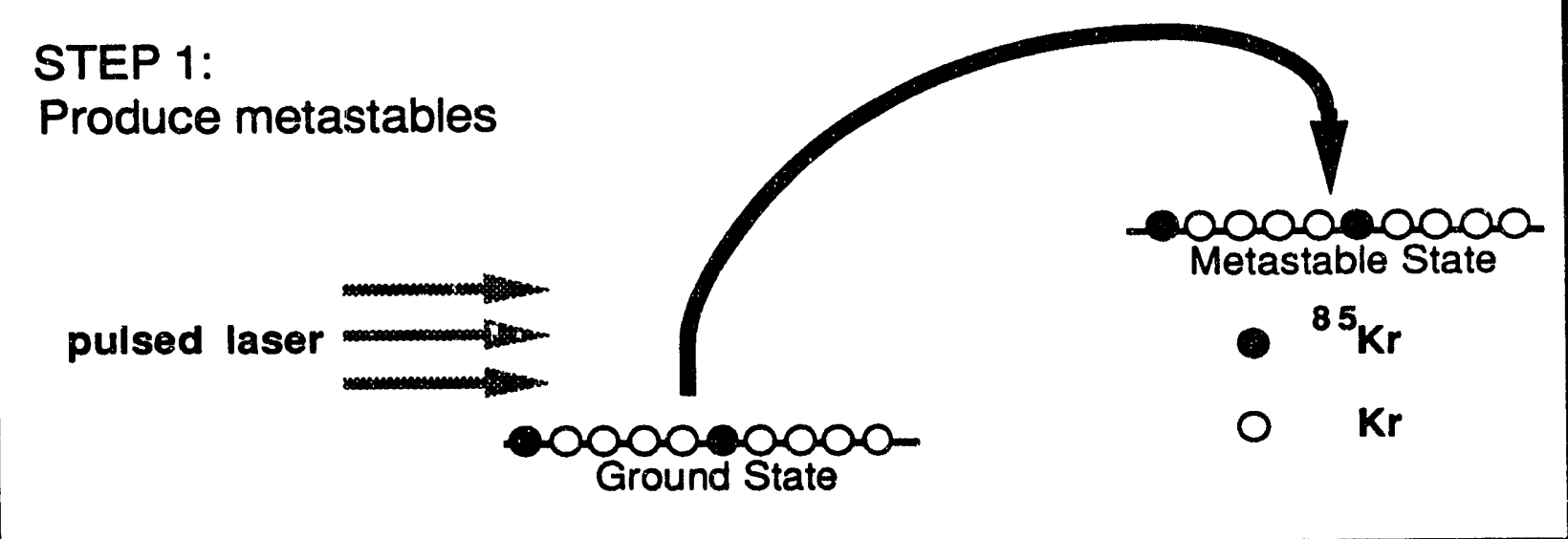

\section{STEP 2:}

Deplete unwanted isotopes

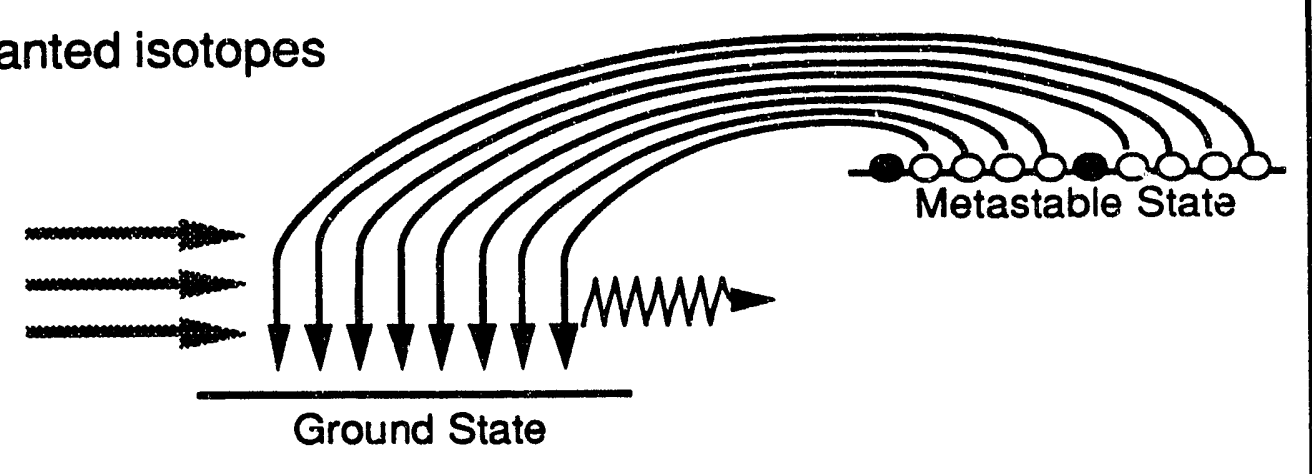

STEP 3:

Measure ${ }^{85} \mathrm{Kr}$

CW laser

EIGURE 1. Measurement of $85 \mathrm{Kr}$ by KILA - The KIllA method uses a three-step process to detect $85 \mathrm{KR}$. In step $1, \mathrm{Kr}$ metastables are produced by two-photon excitation using a pulsed UV laser. In step 2, using a tuned, continuous wave (cw) laser, unwanted stable isotopes of $\mathrm{Kr}$ are depleted by exciting the metastables to an intermediate state that rapidly decays to the ground state

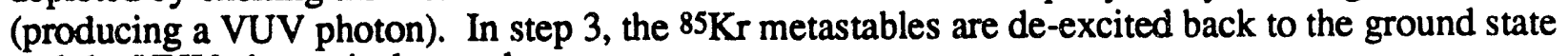
and the VUV photon is detected. 


\section{EXPERIMENTALMETHODS}

The KILA apparatus consists of a sample vacuum chamber, a pulsed dye laser to produce $\mathrm{Kr}$ metastables, a diode laser for monitoring the $\mathrm{Kr}$ metastable population, and a tunable $\mathrm{cw}$ Ti:sapphire laser for de-excitation of metastables.

\section{APPARATUS}

The vacuum chamber, shown in Figure 2, consists of a 4.5-in. (11.4-cm) stainless steel cube. Attached to the cube are a turbo pump (with isolation valve), a connection to a gas-handling manifold, several quartz viewports, and a VUV detector. The cube is fully bakeable and is capable of achieving a background pressure of $<1 \times 10^{-8}$ Torr. The gas-handling manifold is used tn prepare and admit krypton-neon mixtures into the cube. Along one axis of the cube are two quartz viewports, allowing laser beams to transit the central part of the cube's interior. Two other viewports are also attached to the cube, allowing the interior to be viewed from different angles. The VUV detector is contained within a separate chamber and views the cube's volume through a Lyman $\alpha$ filter and a 2-mm-thick $\mathrm{MgF}_{2}$ window, which are, respectively, $17 \%$ and $50 \%$ transparent to the 123.6-nm photons emitted by the krypton metastables. The window permits the detector to be operated at atmospheric pressure while the cube contains a few hundred millitorr of $\mathrm{Kr}$ and Ne. Argon was used as a buffer gas in earlier experiments but was found to be undesirable due to substantial energy transfer from $\mathrm{Kr} 2 \mathrm{p}_{6}$ to $\mathrm{Ar} 1 \mathrm{~s}_{5}$, with resulting unacceptable background fluorescence. No measurable background fluorescence has been attributed to neon.

A small pellet of $\mathrm{Zr}-\mathrm{V}$-Fe getter material was placed in the sample chamber to remove residual reactive gases that might collisionally quench krypton metastables.

\section{DETECTOR}

De-excitation of a $\mathrm{Kr}$ metastable, as shown in Figure 3, produces a VUV photon at $1236 \AA$. In order to measure signals as small as a single photon but with a potential dynamic range of 10 orders of magnitude, the following detector system was developed. The VUV detector (Figure 2) consists of a 14-stage linear-focus photomultiplier tube (PMT) with a 16-mm active area CsI photocathod $\approx$ that is placed $2 \mathrm{~cm}$ from the center of the cube (the region illuminated by the excitation laser). The PMT can detect photons with wavelengths in the region $110-200 \mathrm{~nm}$ and produces a gain of $\sim 5 \times 10^{6}$ when operated at $3 \mathrm{kV}$, which is suficicient to count single photons. To avoid saturation of the phototube by scattered laser light, one dynode out of the dynode chain 


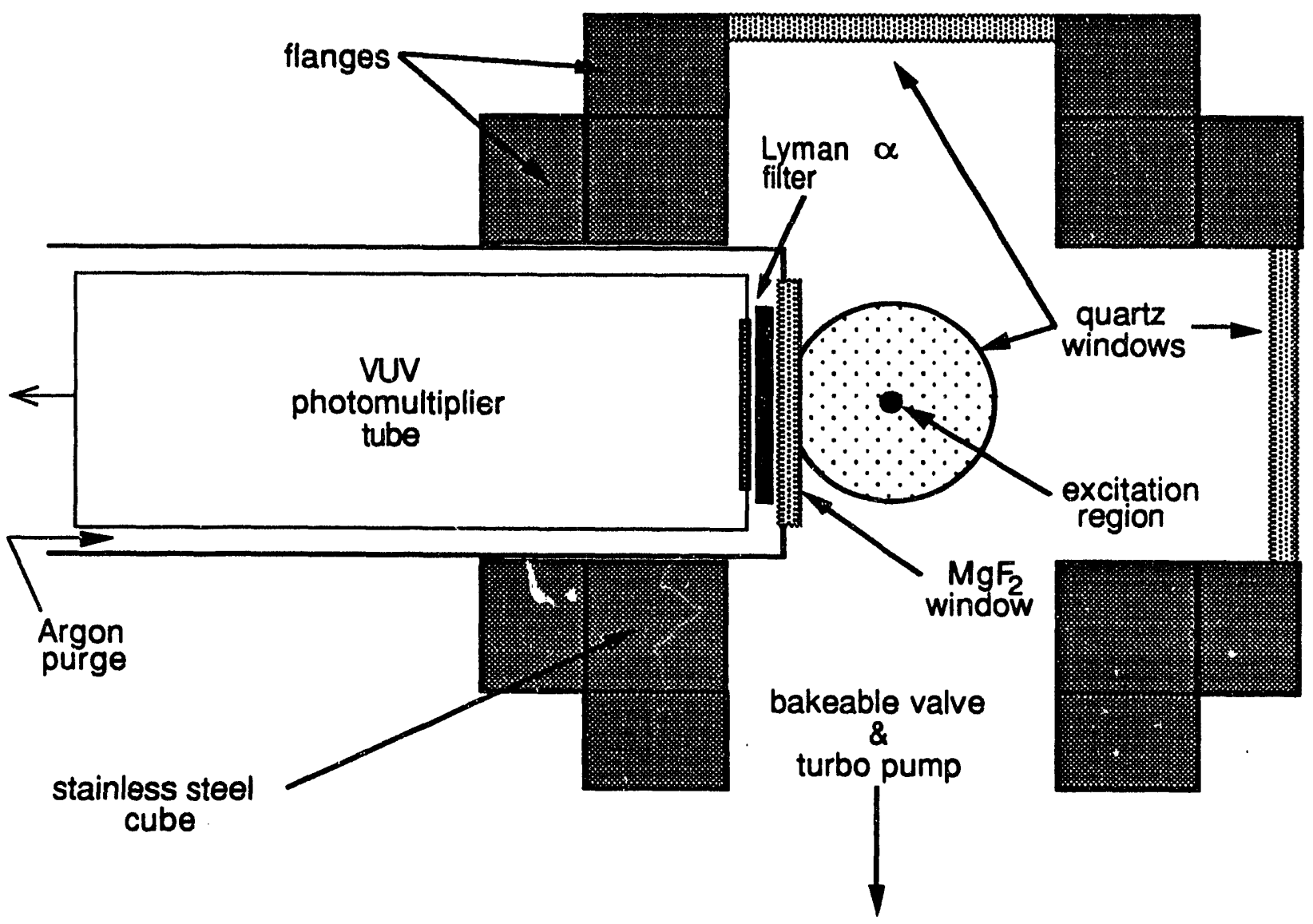

FIGURE 2. Apparatus for detecting VUV fluorescence from $\mathrm{Kr}$ metastables - This apparatus consists of a 4.5 -inch stainless steel ultra-high-vacuum cube to which are actached various quartz windows (for laser propagation), a valve and turbo pump (for evacuating and admitting noble gas mixtures), and a VUV photomultiplier tube. The detector views the excitation volume through a magnesium fluoride window and a Lyman $\alpha$ filter. Not shown is the infrared phototube with which fluorescence from $2 p_{6}$ is measured. 


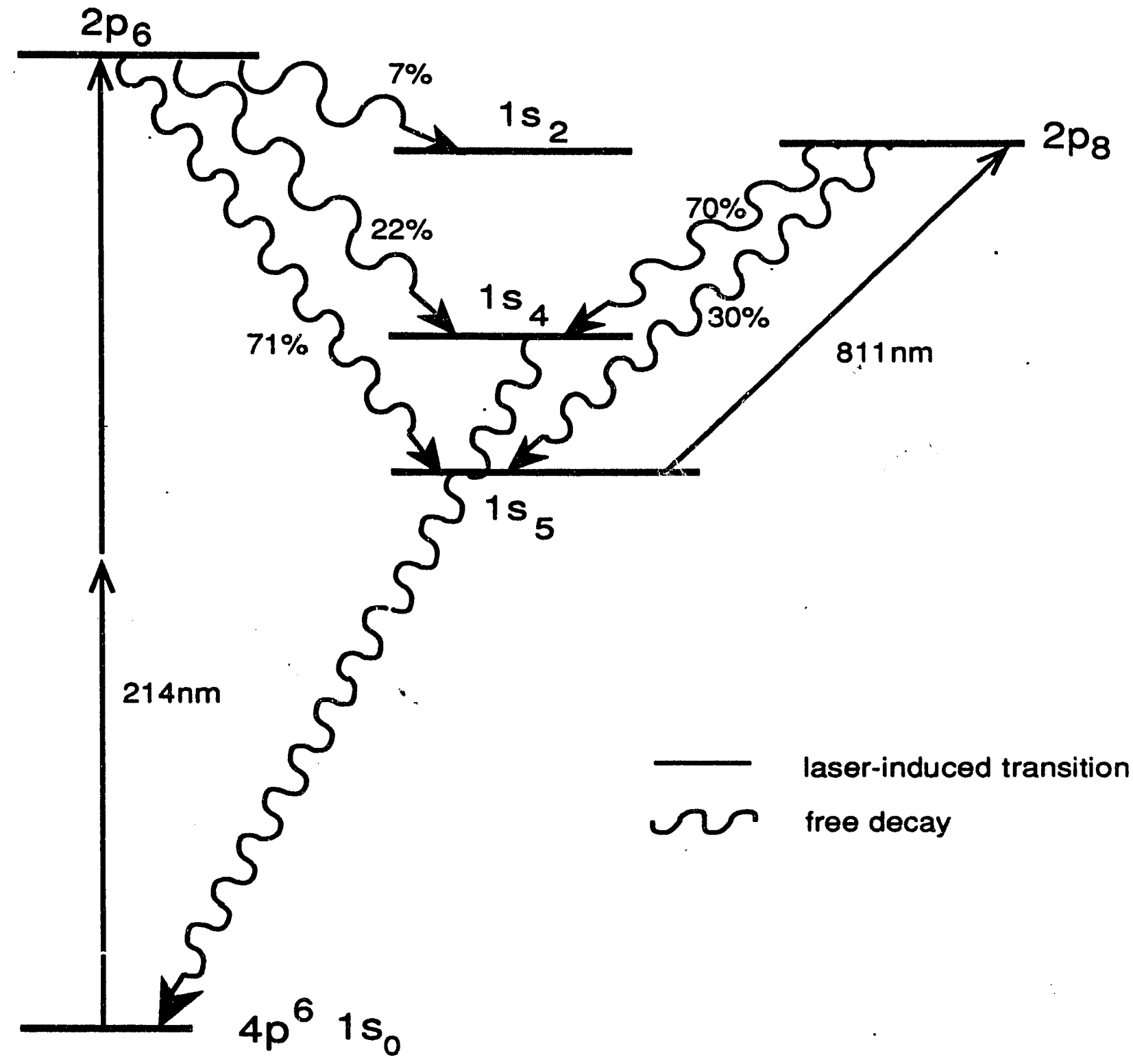

FIGURE 3. Energy-level diagram of excitation/de-excitation scheme - Following two-photon excitation to $2 \mathrm{p}_{6}$, the atom radiatively relaxes to the $1 \mathrm{~s}_{5}$ level with $71 \%$ probability. At this time the $\mathrm{cw}$ laser, tuned to the $1 \mathrm{~s}_{5}-2 \mathrm{p}_{8}$ transition, excites the atom to $2 \mathrm{p}_{8}$. This process is nonisotopically selective since the Doppler width is much broader than the isotope shifts. From $2 \mathrm{p}^{8}$, the atom radiates to the $1 \mathrm{~s}_{4}$ level with $70 \%$ probability. The remaining $30 \%$ radiate back to the $1 \mathrm{~s}_{5}$ level, where they are re-excited. Once in the $1 \mathrm{~s}_{4}$ level, each atom decays to the ground state by emitting a photon with a wavelength of $1236 \AA$. By counting each VUV photon, we count deexcited rnestables. At the pressures used in this experiment, collisional deactivation of energy levels shown here is negligible. 
(typically the second one) was gated from its operating value to roughly half of that value for a few hundred nanoseconds centered around the firing of the excitation laser. Gating was accomplished using a floating $2.5-\mathrm{kV}$ pulsed power supply. During the off phase of operation, the gain is reduced to a point below that at which the output saturates but not low enough so that the gain goes to zero. At zero gain, photoelectrons at the front end of the phototube survive long enough to be swept down the dynode chain and amplified when operating voltages are restored. In order to minimize detection by the phototube of fluorescence at wavelengths other than $1236 \AA$, a Lyman $\alpha$ filter was placed between the $\mathrm{MgF}_{2}$ window of the chamber and the phototube cathode. The filter had a peak transmission of $17.6 \%$ at $1236 \AA$ and a 10 -nm bandpass.

The overall efficiency of this detector is determined by the product of the average viewing solid angle, the transmission of the window, the transmission of the Lyman $\alpha$ filter, the quantum efficiency of the PMT at $123.6 \mathrm{~nm}$, and the fraction of pulses exceeding the discriminator level. We estimated these factors as $4 \%, 50 \%, 17.6 \%, 4 \%$, and $80 \%$, respectively, for an overall efficiency of $\sim 0.01 \%$. There are several changes that could improve the overall efficiency to $1 \%$ or more, including the use of multiple detectors to surround the excitation volume and the use of PMTs with larger active photocathodes.

\section{ELECTRONICS}

The output of the VUV detector PMT was amplified, sent through a discriminator, and then displayed as a function of time using a multi-channel scaler. A Pacific Instruments AD6 amplified the output of the PMT by 100 times and then output NIM-standard pulses for each amplified pulse that exceeded the discriminator level. This level was set for minimumi noise based on measurements of the pulse-height distribution made using a LeCroy Model QVT 3001 multichannel analyzer. Under these conditions, about $80 \%$ of the photoelectrons generated at the PMT cathode produced NIM pulses. The NIM pulses were time-resolved using a LeCroy 3521A multichannel scaler (MCS) with a 110-MHz count rate and $<5$ nanosecond dead time between channels. Even at this count rate, the MCS saturated so that the detector "off" gate-width included early decay of the de-excitation signal. By extrapolating the time-resolved fluorescence back to $t=0$ (pulsed laser fires), we can extract the initial metastable population.

\section{LASERS}

The laser used to produce the $\mathrm{Kr}$ metastables is a single-longitudinal-mode, pulsed, dye laser that is pumped by the 532-nm output of a pulsed, seeded Nd:YAG laser. The dye laser is tunable 
over the range of 600 to $650 \mathrm{~nm}$ and produces 4-nsec pulses with a bandwidth of $\sim 220 \mathrm{MHz}$. The output of the dye laser is amplified by four dye amplifiers (also pumped by the Nd:YAG laser), and the amplified output is frequency doubled and then mixed with the dye laser fundamental to produce UV pulses with an energy of $5 \mathrm{~mJ}$ per pulse at $215 \mathrm{~nm}$. The UV is directed into the sample cell and is tuned to excite the $1 s_{0}-2 p_{6}$ transition in krypton. Tuning of the UV laser is done by maximizing the fluorescence from the $2 \mathrm{p}_{6}-1 \mathrm{~s}_{5}$ transition, as measured using a PMT.

A diode laser is used to probe the metastable population. This laser is locked onto the krypton 1s5 - 2p9 transition at $811 \mathrm{~nm}$ using a krypton discharge reference cell. The diode laser beam is directed into the sample cube coaxial with the UV laser such that the diode laser samples the volume excired by the UV laser. The intensity of the diode laser emerging from the cube is measured using a PMT. Absorption of the 811-nm laser beam indicates that $\mathrm{Kr} 1 \mathrm{~s}_{5}$ metastables are being produced; this signal provides confirmation that the UV laser is properly tuned.

De-excitation to the ground state through $2 \mathrm{p}_{8}$ is accomplished with a Coherent 899-21 Ti:sapphire tunable cw laser pumped by a Coherent Innova 305 argon ion laser. Up to 1 watt of laser light with a root-mean-square(RMS) linewidth of $500 \mathrm{kHz}$ and tunable between 770 and $950 \mathrm{~nm}$ is produced by the Ti:sapphire laser. This de-excitation laser is collinear with, and roughly ten times the diameter of the pulsed 215-nm beam. After passing through the s:mple cell, the laser is chopped and sent through a radiofrequency discharge whose fluorescence is monitored at the laser wavelength using a PMT viewing transverse to the laser propagation axis. The output of the PMT is sent through a lock-in amplifier. By optimizing the signal on the lock-in amplifier, the de-excitation laser is tuned to the $1 \mathrm{~s}_{5}-2 \mathrm{p}_{8}$ resonance. Once tuned to the correct frequency, a laser stabilization circuit using a frequency-stabilized helium-neon laser and a scanning confocal interferometer keeps the de-excitation laser from drifting during the course of the experiment.

\section{NOBLE GASES}

High-purity krypton and neon are used in our experiments. To ensure low impurity levels, getters are used in various parts of the vacuum system for removal of reactive gases (e.g., water vapor) that could deactivate the krypton metastables. 


\section{METASTABLE PRODUCTION}

The KIllA method depends on creating krypton metastables in enough abundance to permit the detection of ${ }^{85} \mathrm{Kr}$ after de-excitation of unwanted isotopes. We have chosen to use the lowestlying metastable(1 $\left.1 \mathrm{~s}_{5}\right)$ state of krypton in order to simplify the spectrometry and laser engineering. Use of krypton in the ground state would require performing all the necessary excitations and measurements in the VUV region of the spectrum - a difficult spectral region in which to build lasers and, in general, in which to work. In contrast, once the metastable state has been reached, there are many accessible transitions in the visible and near-infrared portion of the spectrum that can be used to excite krypton isotopes with good isotopic selectivity. Thus, the critical part of the measurement technique is performed in the visible and near-infrared, and only the initial production of metastables and final detection require working in the UV.

Our method of producing $\mathrm{Kr} 1 \mathrm{ss}$ uses a pulsed laser to two-photon excite krypton to an intermediate state that ra diatively relaxes to the $1 s_{5}$ state. Maximum production of the $\mathrm{Kr} 1 \mathrm{~s}_{5}$ state depends on efficiently producing atoms in the intermediate state for transfer into the $1 \mathrm{~s}_{5}$ state. Also of importance is the partial pressure of krypton in the excitation volume. Higher krypton partial pressures will increase the number of $\mathrm{Kr} 1 \mathrm{~s}_{5}$ produced, but there is a limit set by the rate of transfer for the $1 \mathrm{~s}_{5}$ state excitation between isotopes (i.e., ${ }^{85} \mathrm{Kr}^{*}+{ }^{84} \mathrm{Kr} \rightarrow{ }^{85} \mathrm{Kr}+{ }^{84} \mathrm{Kr}$, where $\mathrm{Kr}^{*}$ is the metastable). Too fast a transfer leads to lost sensitivity or isotopic selectivity. In our method for measuring the ${ }^{85} \mathrm{Kr}$ isotope ratio, there is a tradeoff between sensitivity and isotopic selectivity. From the measured rate for this energy transfer (Brechignac and Vetter 1980), the optimum pressure is $0.1 \mathrm{~m}$ Torr.

The $2 \mathrm{p}_{6}$ state has been determined to be the intermediate state yielding the highest $1 \mathrm{~s} 5$ population. A diode laser tuned to the $1 \mathrm{~s}_{5}-2 \mathrm{pg}$ transition is sent through the sample cell collinear with the pulsed excitation laser. The peak absorption of the diode laser beam was $45 \%$ during metastable production, corresponding to a $0.15 \%$ efficiency in producing $\mathrm{Kr} 1 \mathrm{~s} 5$. The linewidth in this Doppler-free two-photon excitation was $1.3 \mathrm{GHz}$, a factor of three less than the Doppler width, and was due to the dye laser fundamental linewidth of $220 \mathrm{MHz}$.

Finally, in addition to measuring the efficiency for producing krypton $1 \mathrm{ss}_{5}$ metastables, we measured the lifetime of the metastables by fitting the absorption signal and also by time-resolved fluorescence measurements using a multi-channel scaler. Both methods agreed very well and yielded lifetimes of $1.2 \mathrm{msec}$ for the $1 \mathrm{ss}$ level in $1 \mathrm{mTorr}$ krypton and $200 \mathrm{mTorr}$ neon. Previously, we had measured the $\mathrm{Kr} 1 \mathrm{~s}_{5}$ lifetime in 1 Torr argon to be $6 \mathrm{msec}$ (Wacker and 
Cannon 1991). As the neon pressure is increased, the $1 \mathrm{~s}_{5}$ lifetime increases, indicating a loss rate for the $1 \mathrm{~s}_{5}$ state limited by diffusion out of the detection volume. Higher buffer gas pressures would give longer lifetimes but, as will be shown later, the background counts increase with neon pressure due to two-body collisions so that we have a fairly narrow range of buffer gas pressure in which to work. Argon would better serve to confine the metastable population, but the VUV background generated by excitation transfer from krypton to argon renders it undesirable. 


\section{METASTABLE DE-EXCITATION}

In order to count the number of ${ }^{85} \mathrm{Kr}$ metastable atoms by laser-induced fluorescence (LIF), the stable $\mathrm{Kr}$ isotopes, which might interfere with the measurement, must be removed. Ideally, interfering metastables are best removed by putting them into a final state well separated in energy and from which there is no branching fraction back into the original metastable level. An additional requirement is that the metastables be counted as they are removed. The ground state was chosen as the final state and, to coerce the metastable population there, the metastabies were optically excited into the higher-lying $2 \mathrm{p}_{8}$ state, as shown in Figure 3 . The $2 \mathrm{p}_{8}$ energy level has radiative branching fractions of 0.7 into the $1 \mathrm{~s}_{4}$ level, 0.3 into the $1 \mathrm{~s}_{5}$ level, and $<0.01$ into the $1 \mathrm{~s}_{2}$ level (Charg et al. 1980). Exciting the metastables with the Ti:sapphire laser tuned to the 1s5-2p8 transition gives each atom a $70 \%$ chance of radiating to $1 \mathrm{~s}_{4}$, from which it radiates at $1236 \AA$ into the ground state with a branching fraction of 1 . Atoms radiating back into the $1 \mathrm{~s}_{5}$ level are again excited to $2 \mathrm{p}_{8}$.

Overall transfer rates from the $1 \mathrm{~s}_{5}$ to the ground state depend, in part, on the lifetimes of the intermediate states. A 26.5 -nsec radiative lifetime for $2 \mathrm{p}_{8}$ has been measured by Chang, Horiguchi, and Setser (Chang et al. 1980), while we have determined the radiation-trapped $1 \mathrm{~s}_{4}$ lifetime to be $500 \mathrm{nsec}$. The $1 \mathrm{~s}_{2}$-ground state transition is also radiation-trapped with a lifetime slightly shorter than, but comparable to, that of $1 \mathrm{~s}_{4}$ (Matthias et al. 1977). The transfer rate of the metastable population to the ground state, therefore, could be as large as $2 \times 10^{6} \mathrm{sec}^{-1}$ but, in practice, is limited by the $1 \mathrm{~s}_{5}-2 \mathrm{p}_{8}$ excitation rate. This rate depends on de-excitation laser intensity, detuning from the transition peak frequency, and laser linewidth. As will be shown later, the maximum rate attainable is $5 \times 10^{5} \mathrm{sec}^{-1}$ so that the lifetime (ime to decrease by $1 / \mathrm{e}$ ) of the metastable during de-excitation is approximately $2 \mu \mathrm{sec}$. After five de-excitation lifetimes, $>99 \%$ of the original metastable population is in the ground state. By counting the number of photons emitted at $1236 \AA$ from the excitation volume, we know how many metastables have been removed.

Typically, $>20$ de-excitation lifetimes are needed to reduce the metastable population by a factor of $10^{10}$. A typical MCS scan demonstrating a de-excitation rate of $2 \times 10^{5} \mathrm{sec}^{-1}$ and a dynamic range of $>10^{6}$ is shown in Figure 4. The apparent de-excitation rate is, again, a convolution of the $1 \mathrm{~s}_{5}-2 \mathrm{p}_{8}$ excitation rate and the $\mathrm{A}$ coefficients for the $2 \mathrm{p}_{8}-1 \mathrm{~s}_{4}$ and $1 \mathrm{~s}_{4}$-ground state transitions. This de-excitation rate is found by fitting the time-resolved fluorescence at $1236 \AA$ to a single exponential using a linear least-squares method. Due to the large dynamic range of 


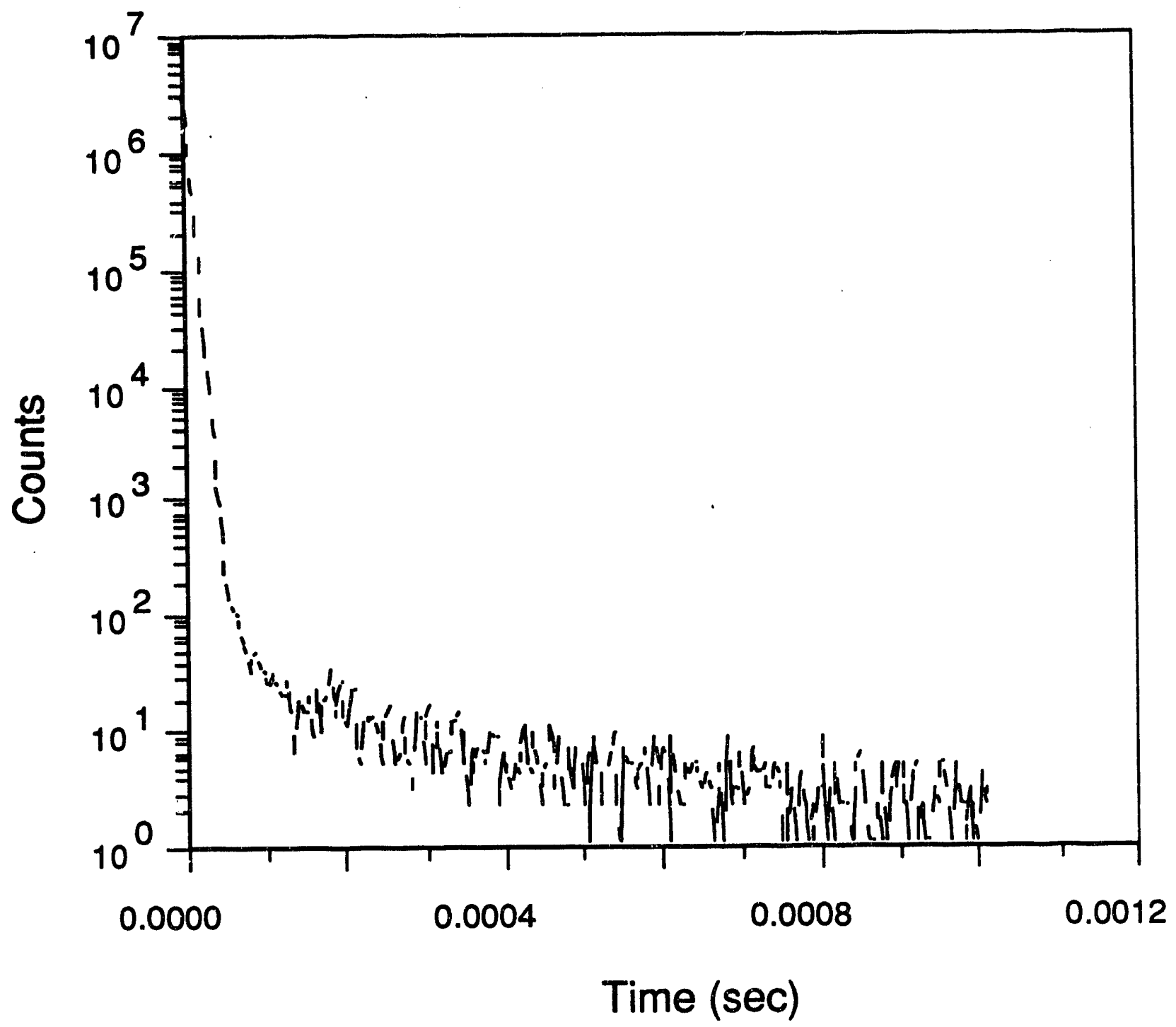

FIGURE 4. Time-resolved VUV fluorescence from $1 \mathrm{~s}_{4}$ level - data taken on $1 \mathrm{mTorr} \mathrm{Kr}$ in 200 mTorr Ne. De-excitation laser tuned to $1 \mathrm{~s}_{5}-2 \mathrm{p}_{8}$ resonance. 
measurement, a linear least-squares fit of the natural log of the data returns values that are within $1 \%$ of those returned by nonlinear least-squares and requires less calculation time. The dependence of the de-excitation rate on buffer gas pressure can be explained by hole-burning in the velocity distribution of the metastable atoms. The linewidth of the Ti:sapphire laser was $500 \mathrm{kHz}$ RMS while the Doppler width of the $1 \mathrm{~s}_{5}-2 \mathrm{p}_{8}$ transition is $500 \mathrm{MHz}$. Only the initial metastable population that lies within this laser linewidth is depleted at the maximum rate of $5 \times 10^{5} \mathrm{sec}^{-1}$. The remainder, outside the laser linewidth, rely on collisions with neighboring atoms to re-orient their velocity vectors to lie within the laser linewidth. The time-resolved decay thus exhibits a clear twocomponent decay at lower buffer gas pressures. For this reason, the apparent de-excitation rate increases drastically with buffer gas pressure until the velocity-changing collision rate is greater than or equal to the optical de-excitation rate. At this point, the de-excitation rate levels off to a value limited by the oscillator strength of the $1 s_{5}-2 \mathrm{p}_{8}$ transition. This effect is shown in Figure 5 .

By defining the background to be the ratio of the late-time signal $(t>100 \mathrm{msec})$ to the early signal $(t<100 \mathrm{msec}$ ), we can plot the variation of this background with buffer gas pressure (Figure 6). The background decreases dramatically as the pressure increases until it reaches a minimum at $\sim 250 \mathrm{~m}$ Torr, at which point it increases at a slow rate. The decrease of the background with pressure is explained by velocity hole-burning as argued above. At higher pressures, the background increases because of the increase in the amplitude of a slower decay component. The slow decay is fluorescence from metastables that diffuse out of the excitation volume and decay via two-body collisions to the $1 s_{4}$ level and subsequently relax to the ground state via emission of a $1236 \AA$ photon. The ultimate signal-to-noise $(\mathrm{S} / \mathrm{N})$ ratio, therefore, depends strongly on buffer gas pressure. Both de-excitation rate and background were independent of krypton pressure over the range 0.1 to $5 \mathrm{mTorr}$. By appropriately tailoring the partial pressure of neon, we obtain the time-resolved metastable population shown in Figure 4. While larger deexcitation rates are attainable, the largest $S / N$ value is obtained at a de-excitation rate of $2 \times 10^{5} \mathrm{sec}^{-1}$.

In order to measure concentrations of ${ }^{85} \mathrm{Kr}$ of $<1$ part in $10^{10}$ with a $S / N$ ratio of unity, the background level must be reduced by three orders of magnitude. The lifetime of the background fluorescence is $0.5 \mathrm{msec}$. Fluorescence lasting this long typically indicates ionic recombination or fluorescence due to collisional deactivation of a metastable level. Electric fields ranging between 2 and $50 \mathrm{~V} / \mathrm{cm}$ were applied to the excitation region with no effect on the intensity or lifetime of the background fluorescence. The independence of this background on the presence of an electric field argues against electron-ion recombination. Subsequent experiments will be directed toward determining the source of the remaining background so that it can be further reduced. 


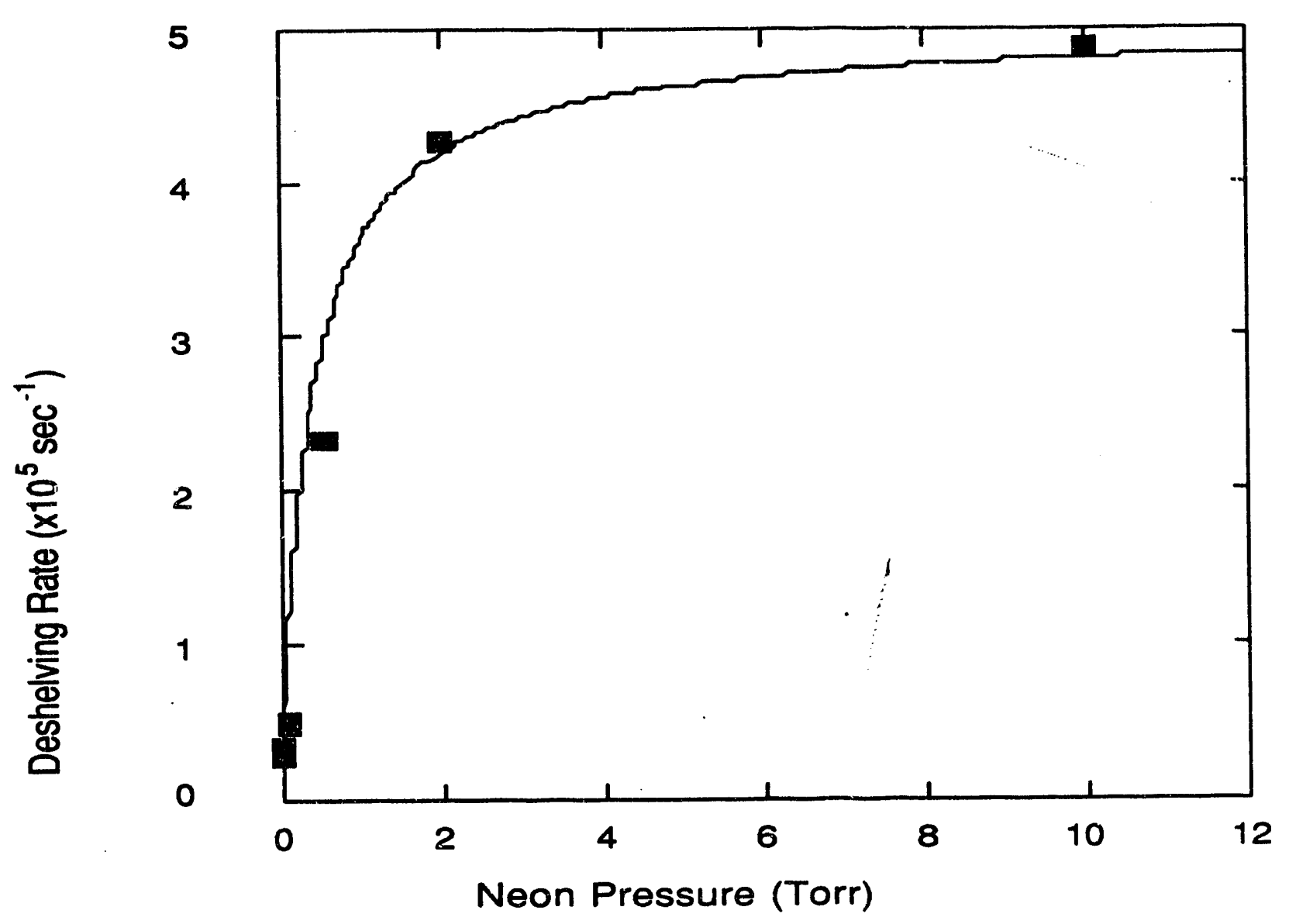

FIGURE 5. Plot of de-excitation rate vs. neon pressure - The decrease at lower buffer gas pressure is due to velocity hole-burning, because laser linewidth is $500 \mathrm{kHz}$ while Doppler width of the $1 \mathrm{~s}_{5}-2 \mathrm{p}_{8}$ transition is $500 \mathrm{Mhz}$. The graph rolls off at higher pressures as velocity-changing collisional rate becomes equal to optical excitation rate. 


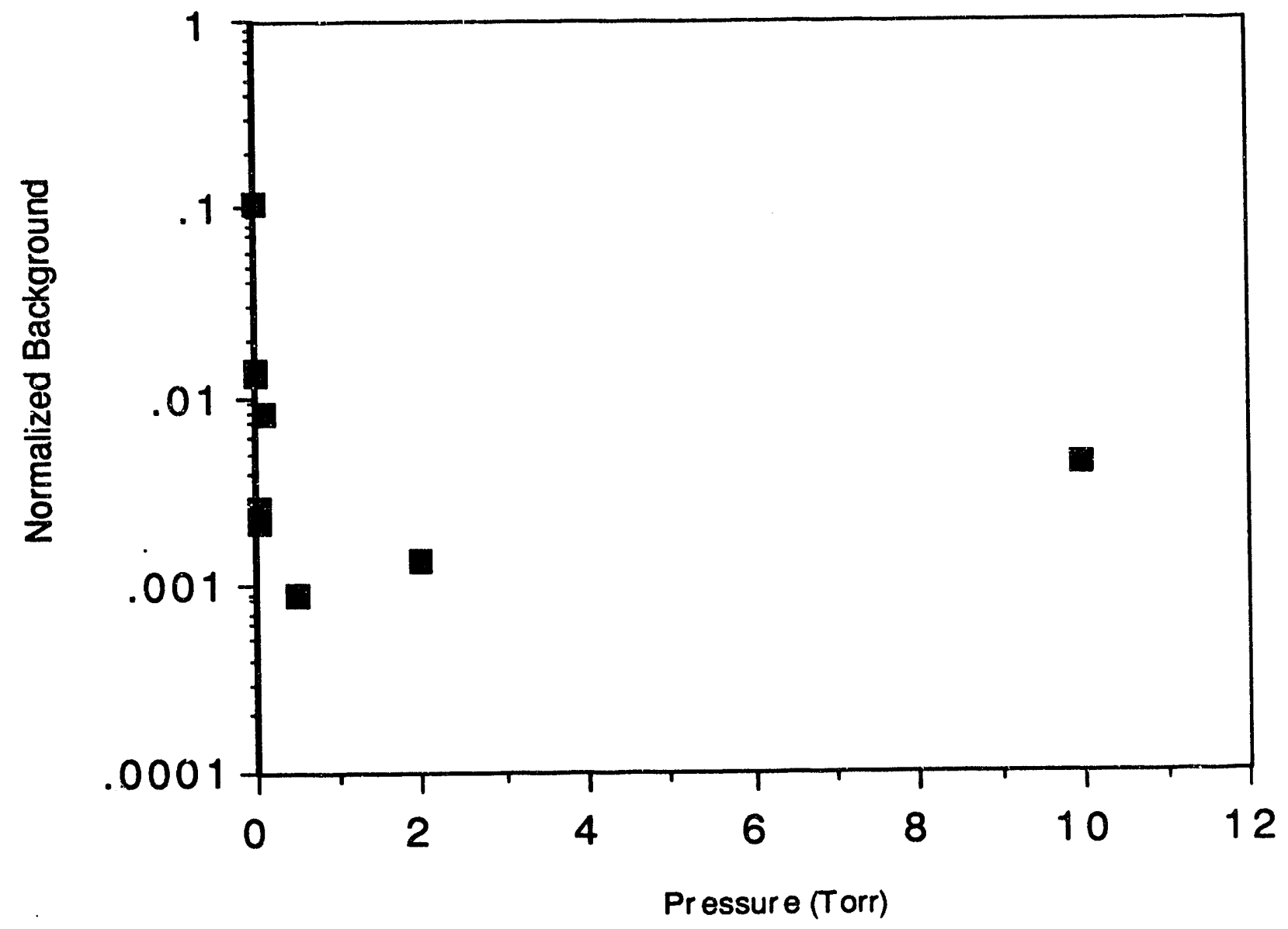

FIGURE 6. Plot of background vs. buffer gas pressure - The background is defined as the ratio of the late time decay $(>100 \mu \mathrm{secs})$ to the early decay $(<100 \mu \mathrm{secs})$. The minimum occurs between 200 and $500 \mathrm{~m}$ Torr of neon. Below this, the background increases due to velocity hole-burning effects as described in text. At higher neon pressures, the background increases because of collisional deactivation to $1 \mathrm{~s}_{4}$ of metastables that diffuse out of the excitation volume. 


\section{CONCLUSIONS}

After demonstrating the production of krypton metastables with the required efficiency by means of two-photon absorption to 2p6, we implemented a VIV detection system with which to investigate preliminary de-excitation rates for $\mathrm{Kr}$ metastables. Non-isotopically selective deexcitation rates were measured to test the feasibility of using LIF on $\mathrm{Kr}$ metastables to eliminate unwanted isotopes and rapidly count ${ }^{85} \mathrm{Kr}$. By exciting the metastables via one-photon absorption to a higher-lying level and monitoring the fluorescence during relaxation to the ground state, we measured de-excitation rates as high as $5 \times 10^{5} \mathrm{sec}^{-1}$. For the largest $\mathrm{S} / \mathrm{N}$ values, a rate of $2 \times 10^{5}$ $\mathrm{sec}^{-1}$ was obtained. The lifetime of the metastables is $1.2 \mathrm{msec}$, while the time required to optically deplete the metastable population by a factor of $1 / \mathrm{e}$ and to detect fluorescence produced by ${ }^{85} \mathrm{Kr}$ is $5 \mu \sec \left(1 /\right.$ de-excitation rate). To reduce the unwanted metastable population by a factor of $10^{10}$ would take $115 \mu \mathrm{sec}$, after which time $91 \%$ of the ${ }^{85} \mathrm{Kr}$ population is still preserved (neglecting losses due to metastability exchange). While a further reduction in background remains to be implemented, LIF and photon counting are shown to yield values consistent for overall development of a laser-based analytical method such as KILA. Short-term goals include characterization of an isotopically selective de-excitation rate on krypton samples of known isotopic abundances. 


\section{REEERENCES}

Brechignac, C., and R. Vetter. 1980. "Measurement of a Metastablility Exchange Cross Section in Krypton." Phys. Rev. A. 22:496.

Chang, R. J. F., H. Horiguchi, and D. W. Setser. 1980. "Radiative Lifetimes and Two-Body Collisional Deactivation Rate Constants in Argon for $\mathrm{Kr}\left(4 p^{55 p)}\right.$ and $\mathrm{Kr}\left(4 p^{55 p}\right.$ ') States." J. Chem. Phys. 73:778.

Matthias, E., R. A. Rosenberg, E. D. Poliakoff, M. G. White, S. T. Lee, and D. A. Shirley. 1977. "Time Resolved VUV Spectroscopy Using Synchrotron Radiation: Fluorescent Lifetimes of Atomic $\mathrm{Kr}$ and Xe*." Chem. Phys. Lett. 52:239.

Wacker, J. F., and B. D. Cannon. 1991. Krypton Metastable Production. PNL,-7886, Pacific Northwest Laboratory, Richland, Washington. 
PNL-8629

UC-713

\section{DISTRIBUTION}

No. of

Copies

\section{OEFSITE}

12 DOE/Office of Scientific and Technical Information

\section{A.L.Boni \\ Savannah River Technology Center P.O.Box 616 \\ Aiken, SC 29802}

\section{L.A. Casey}

DP5.1

U.S. Department of Energy

Washington, DC 20585

T.C.Chapman

Idaho National Engineering Laboratory

P.O.Box 3813

Idaho Falls, ID 83403-3813

C.Chen

Brookhaven National Laboratory

Upton, NY 11973

W. Conaway

Lawrence Livermore National Laboratory

P.O.Box 808

Livermore, CA 94550

P.R.Gathals

Los Alamos National Laboratory

P.O.Box 503

Los Alamos, NM 87545

J.B. Gerardo

Sandia National Laboratories

P.O. Box 5800

Albuquerque, NM 87185
No. of

Copies

J.R.Phillips

Los Alamos National Laboratory

P.O.Box 1663

Los Alamos, NM 87545

J.M. Ramsey

Oak Ridge National Laboratory

P.O. Box 2008

Oak Ridge, TN 37831

\section{M.J.Steindler}

Argonne National Laboratory

9700 S. Cass Ave.

Argonne, IL 60439

\section{ONSITE}

DOE Richland Field Office

R.B. Goranson, A5-90

16 Pacitic Northwest Laboratory

C.A.Whitehead, K3-58

B.D. Cannon, K3-57 (4)

J.F. Wacker, $\quad$ P7-07 (4)

N.A. Wogman, P7-35

Publishing Coordination

Technical Report Files (5) 

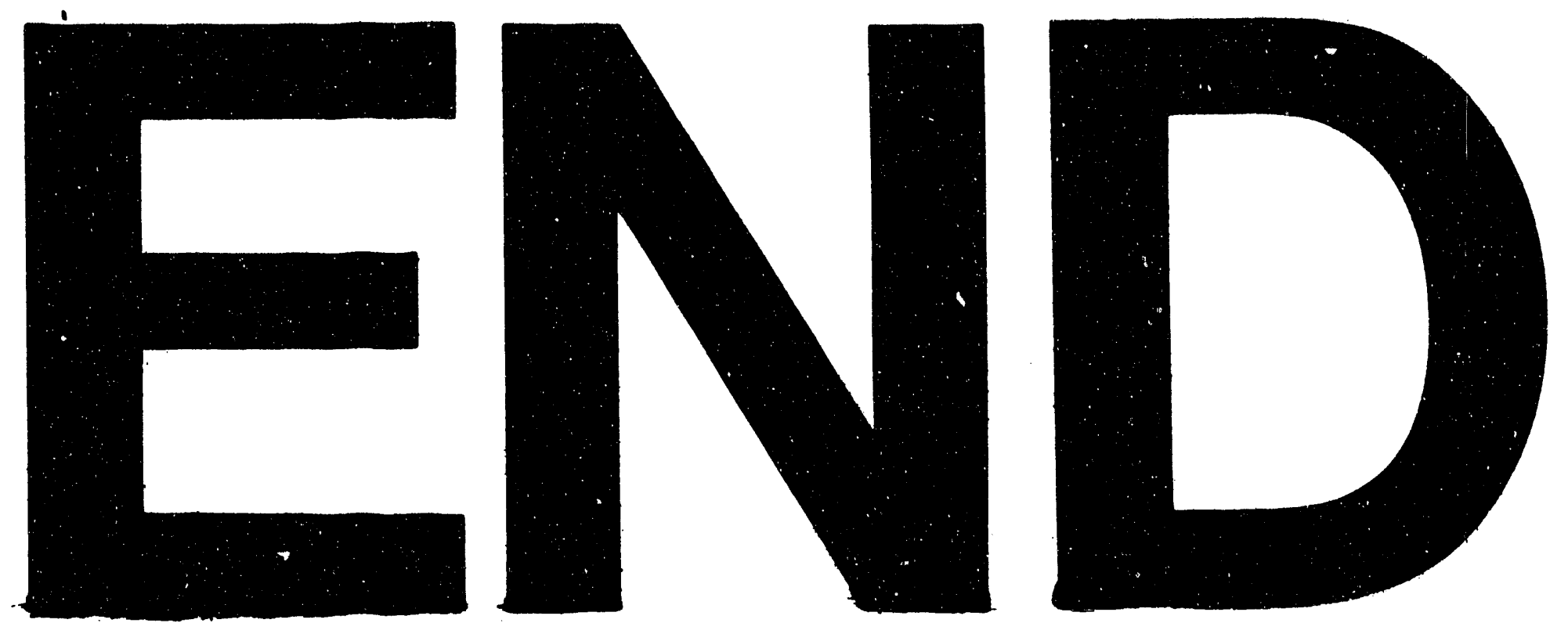

feq
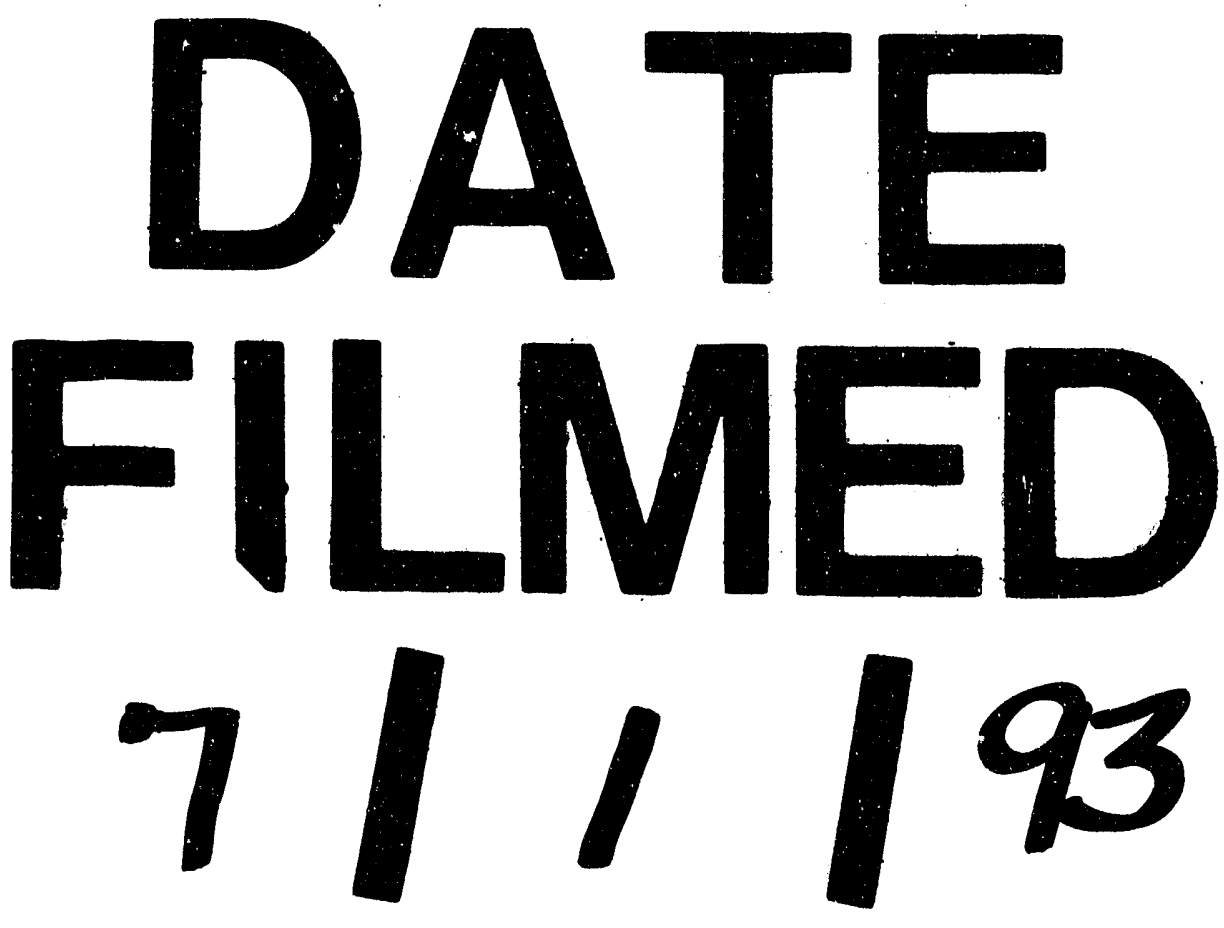

高 
